
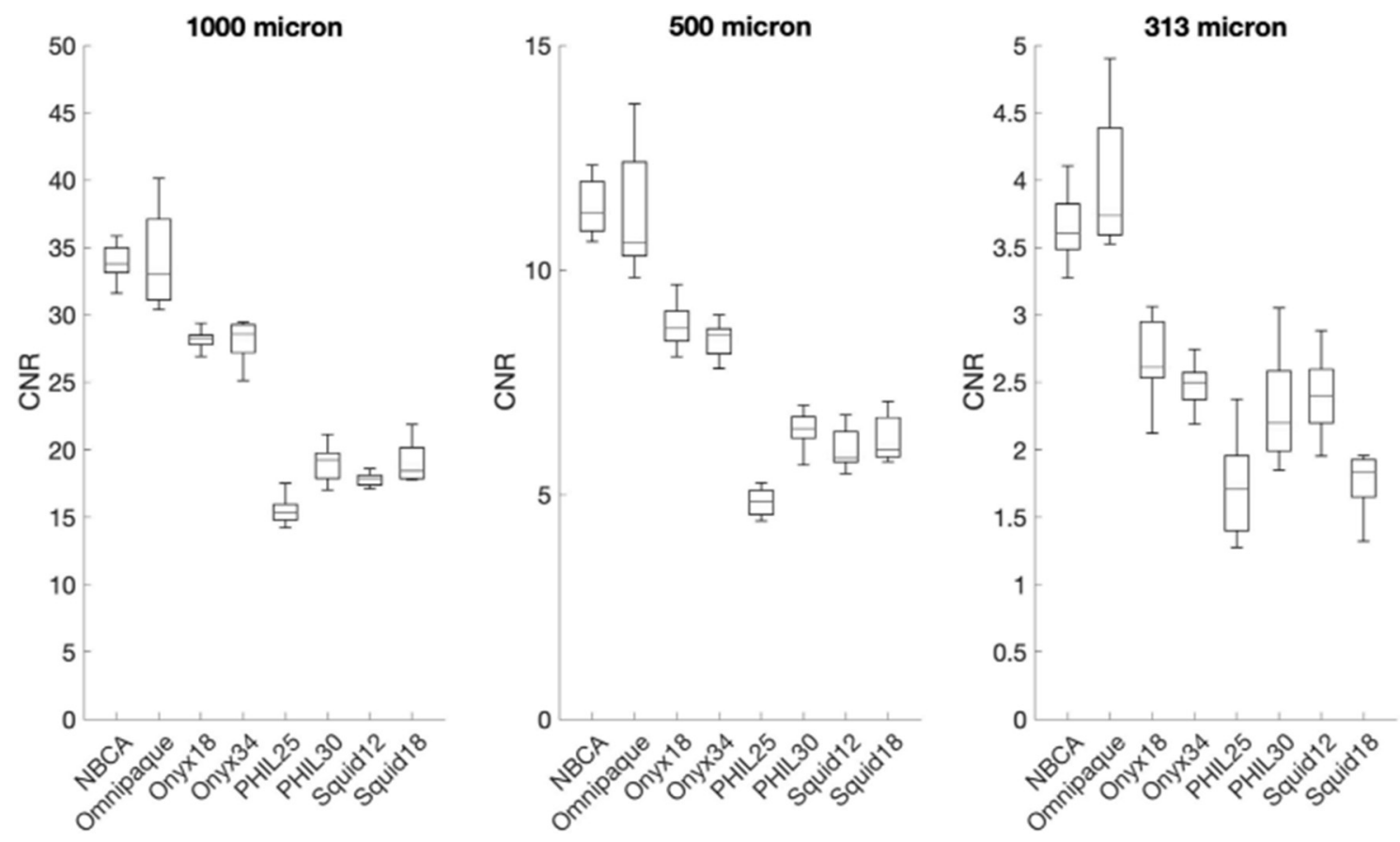

Abstract 0-015 Figure 1

(CNR) and calculated as mean peak signal minus mean background signal divided by the standard deviation of the background signal.

Results Omnipaque 300 and Trufill demonstrated significantly the highest CNR (CNR 11.3-11.4 at 500 micron and CNR $34.1-34.2$ at 1000 micron). Onyx 18 and Onyx 34 (8.5-8.8 at 500 micron and 28.1-28.2 at 1000 micron) both showed significantly higher CNR than all SQUID ${ }^{\oplus}$ and PHIL $^{\oplus}$ concentrations. There was no significant difference between SQUID ${ }^{\circledR} 12$, SQUID ${ }^{\circledast} 18$, and PHIL $^{\oplus} 30$ (CNR 6-6.4 at 500 micron and CNR 17.8-19 at 1000 micron). PHIL $^{\oplus} 25$ (CNR 4.8 at 500 micron and CNR 15.4 at 1000 micron) demonstrated the lowest CNR for all measurements. CNR results were consistent across 1000 and 500 micron tubing sizes with minor variation at 313 micron.

Conclusions There is significant variability in CR for the various LEAs under the RM conditions used in this study. Significantly different CR between most LEAs (except Trufill ${ }^{\circledR}$ ) and iodinated contrast media was evident and should be considered prior to injection. Knowledge and understanding of different visualization performance among various LEAs are important to avoid non-target vessel embolization. Subsequent studies are needed to further establish this method for improving image quality and increase patient's safety during EVT of cerebral AVMs and DAVFs.

Disclosures J. Mason: 1; C; Balt.

\section{0-016 THE DISTRIBUTION AND ROLE OF M1 AND M2 MACROPHAGES IN HEALING OF ANEURYSMS AFTER PLATINUM COIL EMBOLIZATION}

${ }^{1} Z$ Khashim*, ${ }^{1} D$ Daying, ${ }^{1} D$ Yong Hong, ${ }^{2} S$ Herting, ${ }^{1} T$ Caracena, ${ }^{1} D$ Jakaitis, ${ }^{2} D$ Maitland, 'DF Kallmes, 'R Kadirvel. 'Radiology, Mayo Clinic, Rochester, MN; ${ }^{2}$ Biomedical Engineering, Texas AandM University, College Station, TX

10.1136/neurintsurg-2019-SNIS. 16
Introduction Pro-inflammatory coatings of platinum coils have been used in the treatment of intracranial, saccular aneurysms but failed to reduce recurrence rates. Dysregulation of macrophage polarization, in which pro-inflammatory M1 macrophage population fail to transition to an anti-inflammatory M2 macrophage phenotype, is a characteristic feature of poorly healing wounds. In this study, we aimed to evaluate the distribution of M1 and M2 macrophages and to analyze their association with healing in aneurysms embolized by endovascular coiling.

Methods We created 17 elastase-induced aneurysms in female rabbits and subsequently embolized with platinum coils. Aneurysm occlusions were evaluated with angiographic imaging. Aneurysm tissue was harvested at 1, 3, and 6 months for evaluation. Quantification of M1 and M2 macrophage expression was performed by immunofluorescence. Collagen deposition was determined by Masson's trichrome staining technique. Histologic grading of aneurysms was also performed.

Results Angiographic data indicated no significant differences in the geometry of aneurysms between the three treatment-tosacrifice time groups. M1 macrophage expression in aneurysm tissues was highest at 1 month post-treatment and progressively decreased at 3- and 6 months. Expression of M2 macrophages progressively increased at 3 months and 6 months post-treatment. Collagen deposition was highest at 6 months post-treatment. Our data demonstrated a moderate to weak positive relationship between M2 macrophage expression and collagen deposition and total histologic scores and a strongly positive relationship between M2 macrophage expression and total histologic scores at 6-months post-treatment.

Conclusion Our study showed that M2 macrophage expression had a strong positive relationship with total histologic scores at a later stage of healing after endovascular coiling. These findings suggest that interventions aimed at stimulating M2 macrophage expression may improve aneurysm healing after embolization. 

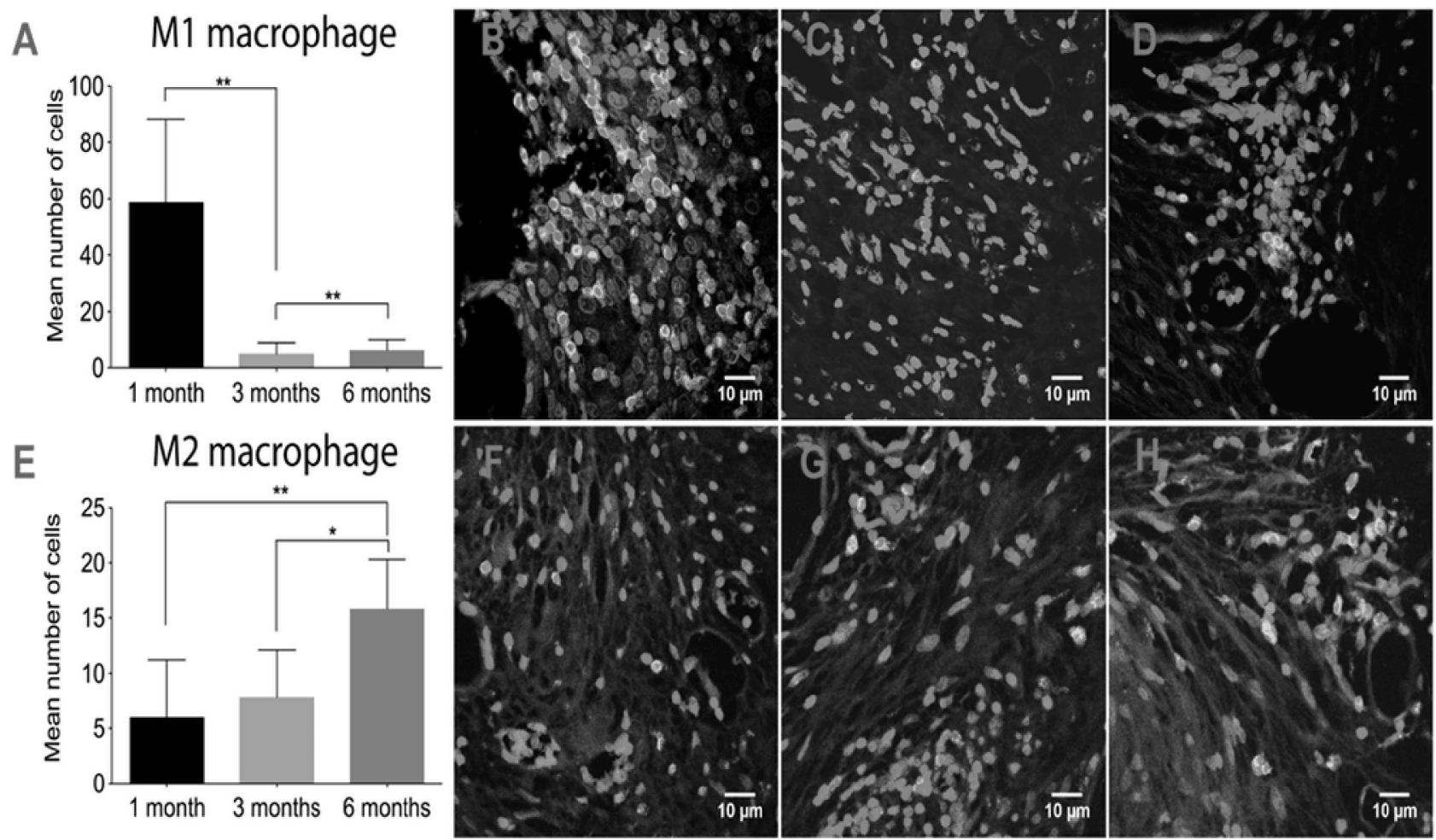

Abstract 0-016 Figure $1 \quad M 1$ and M2 macrophage expression in aneurysm tissues after endovascular coiling for three treatment-to-sacrifice time groups. A) mean number of M1 macrophages; representative confocal microscopy image of M1 macrophages (60X oil magnification) at 1 month (B), 3 months $(C)$ and 6 months (D); E) mean number of M2 macrophages; representative confocal microscopy image of M2 macrophages (60 X oil magnifications) at 1 month (F), 3 months (G) and 6 months $(\mathrm{H}) .{ }^{*} \mathrm{P}<0.05 ;{ }^{* *} \mathrm{P}<0.01 ;{ }^{* * *} \mathrm{P}<0.001$

Disclosures Z. Khashim: None. D. Daying: None. D. Yong Hong: None. S. Herting: None. T. Caracena: None. D. Jakaitis: None. D. Maitland: None. D. F. Kallmes: None. R. Kadirvel: None.

\section{0-017 EDINBURGH'S WEB EXPERIENCE}

N Dobbs*, J Du Plessis, P Keston, J Downer. Edinburgh Department of Clinical Neuroscience, Edinburgh, UK

\subsection{6/neurintsurg-2019-SNIS. 17}

Purpose The Woven EndoBridge (WEB) device has recently gained FDA approval in the United States of America. To increase physician familiarity with the device, its efficacy and safety profile in general usage, we present the Edinburgh case series of WEB usage in both the elective and acute setting.

Materials and methods Data was recorded prospectively on all patients who had an aneurysm treated with the WEB device in Edinburgh between February 2014 and October 2018. The information included patient demographics, aneurysm characteristics, procedural information including the use of antiplatelet medication and the size of WEB used, procedural complications as well as clinical and angiographic follow up at three months and one year.

Results 45 acute and 46 elective cases were identified. Aneurysm sizes ranged from $2.5 \mathrm{~mm}$ to $10.9 \mathrm{~mm}$. Median screening time was 14 minutes. Irrecoverable parent vessel occlusion occurred in $1 \%$ (1/91). WEB protrusion which required stent occurred in 3\% (3/91). There were no intraoperative ruptures. The WEB device was resized in $12 \%$ of cases (11/91). At three months adequate occlusion (complete occlusion or small neck remnant) was achieved in 92\% (68/74), with $8 \%(6 / 74)$ significant remnant, all of which were retreated. Cumulative one year results showed $80 \%$ (33/41) adequate occlusion and $20 \%(8 / 41)$ aneurysm remnant, all of which were retreated with stent assisted coil embolization.

Conclusion The results confirm that the WEB device allows safe, quick and effective treatment of wide necked aneurysms, both ruptured and unruptured, in day to day practice.

Disclosures N. Dobbs: None. J. Du Plessis: None. P. Keston: None. J. Downer: None.

\section{0-018 WEB ANEURYSM TREATMENT: 2-YEARS FOLLOW-UP IN THE 3 EUROPEAN GCP STUDIES (WEBCAST, WEBCAST 2, FRENCH OBSERVATORY)}

'L Pierot*, ${ }^{2} \mathrm{X}$ Barreau, ${ }^{1}$ I Szikora, 'D Herbreteau, 'J Byrne, 'L Spelle. 'Radiology, Hôpital Maison-Blanche, Reims, France; ${ }^{2}$ Neuroradiology, Hôpital Maison-Blanche, Reims, France

\subsection{6/neurintsurg-2019-SNIS.18}

Introduction/Purpose Flow Disruption with WEB is an innovative endovascular approach for wide-neck bifurcation aneurysms that has been evaluated in 3 European Good Clinical Practice Studies: WEBCAST (WEB Clinical Assessment of Intrasaccular Aneurysm), French Observatory, and WEBCAST2. This treatment is associated with a high safety and efficacy at one year. ${ }^{1}$ Long-term anatomical results have been very few evaluated. The present work is presenting the 2-years safety and efficacy results in the 3 GCP studies.

Materials and methods Out of the 168 patients with 169 aneurysms initially included in the 3 GCP studies, 138 patients were clinically evaluated at 2 years and 120 patients with 121 aneurysms were evaluated by vascular imaging. 